\section{PLANT VIRUSES AND VIRUS DISEASES*}

\author{
By F. C. BAWDEN \\ Rothamsted Experimental Station
}

$\mathrm{T}$ HE existence of viruses was first deduced from work done in 1892 on tobacco plants suffering from mosaic, and much of what we now know of these elusive entities has come from further work on this and a few other plant diseases. It is far from certain that this knowledge can safely be applied to the causes of the many diseases, affecting all kinds of animals, higher plants and bacteria, that are now attributed to viruses. These cover a wide range of clinical conditions, and we know for certain of only two features that they have in common ; their causes have neither been seen nor cultivated in vitro. If we wish, we can turn these negative features into what looks like a positive statement, by defining viruses as obligately parasitic pathogens too small to be resolved by microscopes using visible light. Indefinite as this is, it may still prove to be more precise than the facts warrant, for obligate parasitism is always postulated rather than proved, and serious attempts at cultivation have actually been made with very fow viruses. Thus, when we speak of a virus disease, we usually mean merely an infectious disease with an invisible cause. Unless the resolving power of the microscope has some unsuspected significance in defining biological types, this obviously tells us nothing specific about the nature of viruses and might well cover a range of different entities.

This possibility seems increasingly likely when we try to generalize about plant virus diseases, for we find that no statements can be made about such features as symptoms, methods of infection, or distribution of virus in the host, to which there are no exceptions. This is far from conclusive, however, for what a virus does to a plant is as much a property of the plant as of the virus, and the same virus may produce very different effects in different plants. Also, although complete generalizations are impossible, there are some features shared by a number of different virùs diseases, especially those met commonly in Nature.

The effects most frequently caused by viruses are a dwarfing of the host plants and an alteration of the colour and shape of the leaves. Instead of being uniformly dark green, the leaves may bear spots, rings or patches of light green, yellow or white, or they may be generally chlorotic without definite mottling. Deformation may show only as an alteration in the leaf outline, or the laminæ may be so reduced that the leaves consist of little but the main veins ; it may take the form of local hyperplasia, to give unusual outgrowths from the leaves or gall-like proliferations in stems. Symptoms tend to occur more generally over a whole plant than with most fungal or bacterial diseases, for in natural infections it is usual for viruses to spread through the vegetative parts of affected plants. In plants infected experimentally, however, symptoms are often restricted to local lesions, produced by the death of tissues around the entry point. Diagnosis from symptoms is by no means easy, for different viruses may cause almost identical symptoms in the same host, whereas the same virus may produce totally different clinical

* Substance of two lectures at the Royal Institution delivered on * Substance of two
November 21 and 28 . conditions in different hosts. A further complication is that many viruses are unstable and frequently change to give forms that produce different symptoms from those produced by the parent virus. To be recognized, a virus must cause changes in the appearance of some plants; but it need not necessarily cause changes in all susceptible hosts. Indeed, the phenomenon of the carrier-an infected individual showing no symptoms-is common in plants, and such carriers can be of considerable importance as unsuspected sources of infection for intolerant species.

In many virus diseases, three distinct phases can be identified. As a result of virus multiplication at sites of infection, lesions first appear on inoculated leaves. After a few days, the virus passes to the phloem, through which it travels rapidly to distant parts of the plant. It seems to have no autonomous movement, but to travel along with the translocation stream of elaborated food materials, away from tissues actively engaged in photosynthesis and towards regions of active growth. It is because of this, and not because they resist infection, that leaves already fully developed at the time the plant becomes infected rarely show symptoms. Thus results of systemic infection appear on the young, actively growing leaves; the later symptoms of this systemic phase often differ from those first produced, as the disease passes from an acute to a chronic stage. Often both stages are serious diseases; in many potato varieties, for example, leaf-drop-streak is succeeded by severe mosaic. Occasionally, however, the chronic stage is extremely mild, such as in tobacco plants with ring-spot, which recover from an acute necrotic disease and afterwards show few or no symptoms. The virus is present in such plants, but in smaller quantities than during the acute stage. The sequence of three phases is common, but by no means general, and the same virus may give different sequences in different hosts. Potato virus $\mathrm{Y}$, for example, gives local lesions only in one host, local lesions followed by systemic symptoms of two kinds in a second host, whereas in a third it gives no local lesions and systemic symptoms of only one kind.

In addition to altering the external appearance of plants, viruses also produce internal changes. Some of these are simply modifications of normal structures or tissues, such as reduction of the chloroplasts or necrosis of the phloem; but the most characteristic involve the production of new kinds of intracellular inclusion bodies. These are not found in all virus diseases, but their formation appears to be specific to viruses, for similar bodies have not been found either in healthy plants or in those suffering from other kinds of disease. Different viruses give rise to different kinds of inclusion body, and produce them in varying numbers and in different tissues. The most general type is a vacuolar, amœboid-like body found in the cytoplasm, but crystalline and fibrous inclusions also occur in infections with a number of different viruses. At least two viruses give rise to crystalline inclusions in the nuclei. The precise nature of these bodies is still uncertain; but we know that they contain virus, and their production can in part be simulated in vitro. It seems most likely that they are insoluble complexes produced by the viruses combining with some metabolic product of the diseased plants.

Symptomatology without proof of transmissibility is insufficient to assign a particular disease to the virus group, for similar kinds of symptoms can be caused by toxins, deficiencies of mineral nutrients 
and aberrant genes. With hosts that are easily grafted, transmission by grafting is usually the first method tried; for once organic union is established, all viruses that cause systemic symptoms readily pass from infected scions into healthy stocks. Indeed, grafting is the only method of transmission known for many virus diseases, and it has almost become the critical test of a plant virus disease.

Infection occurs only through wounds, but wounds that permit one virus to enter may not permit another. Many viruses are readily transmitted by rubbing healthy leaves with sap from diseased plants, but others are not; some of both these types are transmitted by insects. Several different explanations can be offered for the failure of inoculation to transmit viruses that are readily transmitted by insects. First, some viruses may be able to establish themselves only in deep-seated tissues, such as the phloem, which are not penetrated by ordinary inoculation methods. Secondly, conditions in the expressed sap of some hosts may be such that the viruses are rapidly destroyed or rendered noninfective. Thirdly, the virus content of sap from some diseased plants may be below that required for infection. Thus, although failure to transmit by inoculation is often used as a specific character of a virus, clearly it may equally well be a reflexion of some property of the host.

Insects do not seem to act simply as mechanical carriers of viruses, for no insect vectors are known for the two viruses most easily transmitted by inoculation; and there appear to be specific relationships between insects and the viruses they transmit. Individual viruses are usually transmitted by only a few related species of insect and not by others, though these may have similar feeding habits and be vectors of other viruses. Vectors are usually insects with sucking mouth-parts; the most important are aphides, leaf-hoppers, white-fly and thrips. Two main types of behaviour in the insect have been distinguished. Vectors of one type of virus can infect healthy plants immediately after feeding for a short time on a diseased plant, and these usually cease to be infective within a few hours. After feeding on diseased plants, vectors of the other type cannot infect healthy plants for some time, which varies from minutes to days with different viruses, and such vectors remain infective for long periods, often for their whole lives. Some workers believe that viruses of the second type multiply in the insects. There is no obvious reason why they should not, and the theory would explain some of the now puzzling features of the behaviour of these viruses; but there is no conclusive evidence that insects ever contain more virus than they acquire while feeding on infected plants. Studies on the virus causing dwarf disease of rice supplies the best circumstantial ovidence for multiplication. This virus is unique in being the only one known to pass from infective adults through eggs to their progeny. Progeny up to the seventh generation have once been found to be infective and from one infected egg the progeny have infected more than 1,000 plants. This is regarded by some workers as 'overwhelming' evidence for multiplication, as they consider that the quantity of virus in the original eggs could not have been onough to give all the infections. But is this so ? If the virus multiplied in the insects to anything like the extent it does in plants, then there would be no reeson why the progeny should not continue to be infective indefinitely, and infect as many plants as they feed on. We know nothing of the size of this virus, but if it is of the same order as other plant viruses the sizes of which are approximately known, then 1,000 particles would weigh less than $10^{-14} \mathrm{gm}$., and many times this quantity could surely be contained in a leaf-hopper's egg without difficulty.

Transmission of some viruses has been achieved by linking diseased and healthy plants with the parasite dodder (Cuscuta sp.). This novel method of transmission promises to be valuable in extending the host ranges of some viruses to plants more favourable for study than those in which the viruses occur naturally. One of the greatest differences between individual viruses lies in the numbers of different plants they can attack. Some are known to infect hundreds of plant species, belonging to many different families and orders ; others have been transmitted to only a few closely related species. This difference may be apparent rather than real, for viruses transmissible only by grafting or by insects will normally have host ranges restricted to plants which can be intergrafted or which can act as food plants for the insect vector.

For more than forty years, work on plant viruses was largely concerned with symptoms, transmission and host ranges. It showed that viruses could multiply and alter, and produced few results conflicting with the generally accepted conclusion that they were small organisms, essentially similar to bacteria. There were opposers of this, usually from among those studying tobacco mosaic virus, but they could offer nothing definite to support their alternative views. The intensive study during the last ten years of the viruses in vitro has led to results that necessitate considerable modification of the earlier views. They do not, however, justify the sweeping conclusions implied by such facile phrases as 'lifeless molecules', which are increasingly applied to viruses.

What has been achieved is the successful application of the techniques of protein chemistry to the purification of a dozen or so viruses. This has shown us that the particles of these viruses are not organized cellularly like organisms, and that in many ways they resemble constituent parts of organisms rather than whole organisms. They can bo obtained in forms chemically much simpler than bacteria, free from diffusible components, and with a much greater regularity of internal structure than is usual with organisms. The viruses so far purified have all been obtained in the same chemical form, as nucleoproteins. They all contain nucleic acid of the ribose type, but the proportion of nucleic acid to protein varies with the individual viruses. It is far too early to conclude that all plant viruses are essentially nucleoproteins; but we can say that it will be a major discovery if one is found to be anything else, for those already purified cover a diversity of types, some known to be insect-transmitted and others not. They range from potato virus $\mathrm{Y}$, which denatures and loses infectivity within a few days, to tobacco mosaic virus, which remains stable for years. Stability as a native protein, however, is not always the same thing as stability as a virus; the infectivity of preparations of any of these viruses can be destroyed by some treatments that have no appreciable effects on the physical, chemical and serological properties.

The shape of the particle is responsible for some of the most striking differences between the properties of preparations of different viruses. Solutions of purified tobacco mosaic virus, and of potato viruses $\mathrm{X}$ and $\mathrm{Y}$, show phenomena characteristic of greatly 
elongated particles; they are anomalous in all their physical properties and are polydisperse. No true crystals have been prepared from these, but dilute solutions show anisotropy of flow strongly, and concentrated solutions are liquid crystalline. X-ray studies of solutions of tobacco mosaic virus have demonstrated a regularity of structure previously unsuspected in fluids, for the particles are arranged equidistant from one another so that the available space is filled uniformly. When mixed with their antisera, these rod-shaped virus particles precipitate almost immodiately, giving bulky, fluffy precipitates resembling those produced by bacterial flagellar antigens.

Solutions of bushy stunt and tobacco necrosis viruses behave very differently and show none of the anomalous properties characteristic of elongated particles. By suitable treatments they can be induced to crystallize in forms characteristic of the individual virus. When mixed with their antisera, they precipitate more slowly than the rod-shaped viruses and, as might be expected with spherical particles, pack more closely to give dense, granular precipitates resembling those produced by somatic antigens.

What is the relationship between these isolated nucleoproteins, which in laboratory work behave much like preparations of other proteins, and the viruses as they occur in the plant ? There is enough evidence now to show that these proteins are the viruses in the sense that they can initiate infection. Nevertheless, it would be premature to assume that, while active in the host plant, the viruses are chemically so simple as analysis of the purified preparations suggests. During the course of isolation, many materials are discarded as impurities ; most of these are certainly constituents of the normal host, but some may well be specific products of virus activity. Any such are clearly not essential for infectivity ; but if the virus were organized cellularly, they would be retained within a cell wall and would be accepted as integral parts of the virus, which would immediately look a much more complex body than does our naked protein particle.

In the absence of specific tests for any product of virus activity, we have no positive evidence for their occurrence in plants, but evidence from various sources suggests that purification may be altering the viruses. Purified preparations of tobacco mosaic virus, for example, contain particles about $15 \mathrm{~m} \mu$ wide but varying in length from less than $100 \mathrm{~m} \mu$ to more than $1,000 \mathrm{~m} \mu$. There is nothing to show that the greatly elongated particles occur in the plant, and much to suggest that they are produced by the linear aggregation of small particles during the course of preparation. By taking suitable precautions, solutions of tobacco mosaic virus can be made that show little or no anisotropy of flow and behave serologically more like somatic antigens; but these are unstable and readily change into anisotropic solutions with serological behaviour characteristic of flagellar antigens. This change seems to be connected with the removal of other material from the small nucleoprotein particles, which then join together end-to-end. The change in size and shape may explain the failure to produce true crystals of this virus in vitro, though they occur abundantly in infected plants.

We know also that the purified virus readily combines with other proteins such as trypsin and ribonuclease, and that these can be removed again without affecting infectivity. May not similar com- binations occur within the host, and be responsible for converting this nucleoprotein into a functioning system capable of multiplication and of the activities of which the results are so obvious?

In addition to the changes produced by purification, there is other evidence that virus does occur in the plant in forms with different pro. perties from those of the purified nucleoproteins. Until recently, all laboratory work on plant viruses was done with the sap that is expressed from macerated infected leaves. This was thought to contain all the virus in the plant, for washing the fibrous residues gives little extra virus. However, these residues actually contain as much virus as does the sap; but normally this is insoluble, probably because it is combined with other substances, and special treatments are needed to get it into solution. It is possible that this insoluble virus is the biologically active system, whereas that free in the sap may be merely excess virus functioning as a mobile source of infection for other cells. We know so little about the multiplication of viruses, and of their activities within the host, that at present we must suspend judgment. But it is probably safest to regard the nucleoproteins as the chemical minima-equivalent to reproductive organs or embryonic viruses - which develop into working entities only when placed in an environment containing the materials or enzyme systems they lack in their purified state.

\section{CHEMISTRY AT THE OLDER UNIVERSITIES OF BRITAIN DURING THE EIGHTEENTH CENTURY \\ By ARCHIBALD CLOW \\ University of Aberdeen}

TN 1814 Sir John Sinclair, president of the Board of Agriculture, wrote :

"At present there are a greater number of intelligent practical chemists in Scotland, in proportion to the population, than perhaps in any other country in the world" (J. Sinclair, "General Report", App. 2, p. 307).

In the light of this rather startling assertion, it is instructive to analyse the development of chemistry in the universities of Scotland during the preceding hundred years, and to compare it with developments farther south. In Great Britain there are five universities to consider: in England, Oxford and Cambridge; in Scotland, Edinburgh, Glasgow and Aberdeen. There was no profession of chemistry at St. Andrews until at a later date.

While alchemy yet held the field, the universities of Scotland remained aloof from the flux of gold and elixir making, but at the end of the seventeenth century the Surgeons' Incorporation in Edinburgh established a laboratory where apprentice apothecaries received a chemical training. The instigator of this pioneer development was Alexander Monteith. He was thus a contemporary of Sir Isaac Newton (1642-1727), who in Cambridge was still studying Boyle's method of gold-making during 1690-93, perhaps not without hope of practical application, since within a few years he was appointed Warden of the Mint.

In the early years of the eighteenth century, the Town Council of Edinburgh decided to appoint a 